\title{
Distance Two Surjective Labelling of Paths and Interval Graphs
}

\author{
Sk Amanathulla $\mathbb{D}^{1},{ }^{1}$ G. Muhiuddin $\mathbb{D}^{2},{ }^{2}$ D. Al-Kadi, ${ }^{3}$ and Madhumangal Pal $\mathbb{}^{4}$ \\ ${ }^{1}$ Department of Mathematics, Raghunathpur College, Raghunathpur 723101, India \\ ${ }^{2}$ Department of Mathematics, University of Tabuk, Tabuk 71491, Saudi Arabia \\ ${ }^{3}$ Department of Mathematics and Statistic, College of Science, Taif University, P. O. Box 11099, Taif 21944, Saudi Arabia \\ ${ }^{4}$ Department of Applied Mathematics with Oceanology and Computer Programming, Vidyasagar University, \\ Midnapore 721102, India
}

Correspondence should be addressed to G. Muhiuddin; chishtygm@gmail.com

Received 18 March 2021; Accepted 28 May 2021; Published 7 June 2021

Academic Editor: Juan L. G. Guirao

Copyright (C) 2021 Sk Amanathulla et al. This is an open access article distributed under the Creative Commons Attribution License, which permits unrestricted use, distribution, and reproduction in any medium, provided the original work is properly cited.

\begin{abstract}
Graph labelling problem has been broadly studied for a long period for its applications, especially in frequency assignment in (mobile) communication system, $X$-ray crystallography, circuit design, etc. Nowadays, surjective $L(2,1)$-labelling is a wellstudied problem. Motivated from the $L(2,1)$-labelling problem and the importance of surjective $L(2,1)$-labelling problem, we consider surjective $L(2,1)$-labelling (SL21-labelling) problems for paths and interval graphs. For any graph $G=(V, E)$, an SL21-labelling is a mapping $\varphi: V \longrightarrow\{1,2, \ldots, n\}$ so that, for every pair of nodes $u$ and $v$, if $d(u, v)=1$, then $|\varphi(u)-\varphi(v)| \geq 2$; and if $d(u, v)=2$, then $|\varphi(u)-\varphi(v)| \geq 1$, and every label $1,2, \ldots, n$ is used exactly once, where $d(u, v)$ represents the distance between the nodes $u$ and $v$, and $n$ is the number of nodes of graph $G$. In the present article, it is proved that any path $P_{n}$ can be surjectively $L(2,1)$-labelled if $n \geq 4$, and it is also proved that any interval graph (IG) $G$ having $n$ nodes and degree $\Delta>2$ can be surjectively $L(2,1)$-labelled if $n=3 \Delta-1$. Also, we have designed two efficient algorithms for surjective $L(2,1)$-labelling of paths and interval graphs. The results regarding both paths and interval graphs are the first result for surjective $L(2,1)$-labelling.
\end{abstract}

\section{Introduction}

The frequency assignment problem is bottomed from the problems of distance labelling of graph. In 1992, $L(2,1)$-labelling was invented by Griggs and Yeh [1] in conjunction with channel assigning problem in a multihop radio network.

For any graph $G=(V, E)$, an $L(2,1)$-labelling is a mapping $\varphi: V(G) \longrightarrow\{1,2, \ldots$,$\} , so that |\varphi(u)-\varphi(v)| \geq 2$ if $d(u, v)=1$ and $|\varphi(u)-\varphi(v)| \geq 1$ if $d(u, v)=2$. The span of $L(2,1)$-labelling of $G$ is $\max \{\varphi(v): v \in V\}$. The $L(2,1)$-labelling number $\lambda_{2,1}(G)$ of $G$ is the smallest natural number $p$ so that $G$ has an $L(2,1)$-labelling of span $p$.

A surjective $L(2,1)$-labelling of $G=(V, E)$ is a mapping $\varphi: V \longrightarrow\{1,2, \ldots, n\} \quad$ so that $|\varphi(u)-\varphi(v)| \geq 2$ when $d(u, v)=1$ and $|\varphi(u)-\varphi(v)| \geq 1$ when $d(u, v)=2$, and it requires that each label, $\{1,2, \ldots, n\}$, be used only once, where $n$ is the number of nodes of $G$.
In Figure 1, we have shown an $L(2,1)$-labelling of a path with 5 nodes and Figure 2 shows SL21-labelling of the same graph. In Figure 1, identical label is used several times but in Figure 2 the labels 1 to 5 are used only once. So, in SL21-labelling, there is a more complex task compared to $L(2,1)$-labelling.

In 1994, Sakai has proved some results regarding distance two labelling of chordal graph. Later, in 2007, Bertossi and Bonuccelli have studied approximate $L\left(\delta_{1}, \delta_{2}, \ldots, \delta_{t}\right)$-coloring of trees and interval graphs. Amanathulla and Pal have studied a lot of problems regarding labelling of graphs, like $L(3,2,1)$-labelling problems on permutation graphs [2], $L\left(h_{1}, h_{2}, \ldots, h_{m}\right)$-labelling problems on interval graphs [3], $L\left(h_{1}, h_{2}, \ldots, h_{m}\right)$-labelling problems on circular-arc graphs $[4], L(1,1,1)$ - and $L(1,1,1,1)$-labelling problems of square of paths [5], and $L(3,1,1)$-labelling numbers of squares of paths, complete graphs, and complete bipartite graphs [6]. In 2019, Berhe 


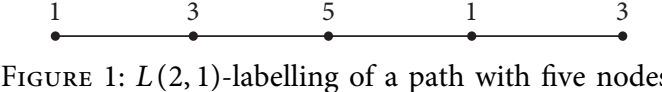

$\begin{array}{lllll}5 & 3 & 1 & 4 & 2\end{array}$

Figure 2: Surjective $L(2,1)$-labelling of a path with five nodes.

and Wang have studied computation of certain topological coindices of graphene sheet and $\mathrm{C} 4 \mathrm{C} 8(\mathrm{~S})$ nanotubes and nanotorus [7]; also, Goyal et al. [8] have studied new composition of graphs and their Wiener indices. Hosamani et al. [9] have studied graphs with equal dominating and c-dominating energy. Very recently, B. M. Gurevich has published one paper regarding classes of infinite loaded graphs with randomly deleted edges [10] and Ranjini et al. have studied degree sequence of graph operator for some standard graphs [11]. For general graph, $\lambda_{2,1}(G) \geq \Delta+1$. In 1992, Griggs et al. showed that $\lambda_{2,1}(G) \leq \Delta^{2}+2 \Delta$ and have proposed a conjecture [1].

In 1993, Jonas [12] has shown that $\lambda_{2,1}(G) \leq \Delta^{2}+2 \Delta-4$. Chang et al. [13] have showed $\lambda_{2,1}(G) \leq \Delta^{2}+\Delta$. Král' and Skrekovski [14] proved that $\lambda_{2,1}(G) \leq \Delta^{2}+\Delta-1$ and they further improved it to $\lambda_{2,1}(G) \leq \Delta^{2}+\Delta-2$ [15].

Different bounds for $\lambda_{h_{1}, h_{2}, \ldots, h_{n}}(G)$ were obtained for different classes of graphs. Some results regarding upper bound of $L\left(h_{1}, h_{2}, \ldots, h_{n}\right)$-labelling are shown in Table 1.

In [37], Lingscheit et al. investigated minimal and surjective labelling for path, cycle, complete graph, caterpillar, and complete bipartite graph. They have shown that $P_{n}$ can be surjectively $L(3,2,1)$-labelled when $n \geq 7$. Very recently, Amanathulla and Pal have studied SL21-labelling of cycle and circular-arc graph (CAG) and obtained good results for it [38].

$L(2,1)$-labelling of graphs is a rapidly studied problem for its applications in various fields, especially in channel assignment in radio network. In $L(2,1)$-labelling, although there is a light chance to overlap the frequencies in radio network, it cannot be neglected, but in SL21-labelling there is no chance to overlap the frequencies, as in this case the labels are distinct. For this reason, in the recent year, SL21-labelling of graph has become a well-studied problem due to its applications. This motivates us to consider SL21-labelling of paths and IGs. Recently, many researchers applied various related concepts on graphs in different aspects (see, e.g., [39-43]).

In the present article, it is shown that any path $P_{n}$ is surjectively labelled by $L(2,1)$-labelling if $n \geq 4$ and it also shown that any IG having $n$ nodes can be surjectively $L(2,1)$-labelled if $n=3 \Delta-1$. Two polynomial time algorithms are also established to label a path and an IG by SL21-labelling.

The remainder of this article is organized as follows: in Section 2, some notations and preliminary definitions are given. In Section 3, SL21-labelling of path has been presented. In Section 4, SL21-labelling of IG is investigated. The last section presents concluding remarks.
TABLE 1: Different types of graphs and their upper bounds.

\begin{tabular}{|c|c|}
\hline Graphs & $L(h, k)$-labelling numbers \\
\hline General graphs & $\begin{array}{c}0 \leq \lambda_{0,1} \leq \Delta^{2}-\Delta[16] \\
\Delta \leq \lambda_{1,1} \leq \Delta^{2}[17] \\
\Delta+1 \leq \lambda_{2,1} \leq \Delta^{2}+\Delta-2[1,15] \\
\lambda_{3,2,1}(G) \leq \Delta^{3}+2 \Delta[18] \\
\lambda_{4,3,2,1}(G) \leq \Delta^{3}+2 \Delta^{2}+6 \Delta[19]\end{array}$ \\
\hline Paths & $\begin{array}{c}\lambda_{0,1}\left(P_{n}\right)=0 \text { or } 1[20] \\
\lambda_{1,1}\left(P_{n}\right)=1 \text { or } 2[21] \\
\lambda_{2,1}\left(P_{n}\right)=2,3 \text { or } 4[1] \\
\lambda_{3,2,1}\left(P_{n}\right)=0,3,5,6 \text { or } 7[22] \\
\text { For } d \geq 4, \lambda_{d, 2,1}\left(P_{n}\right)=0, d, d+2 \text { or } d+4[22] \\
\text { For } n \geq 2 \lambda_{4,3,2,1}\left(P_{n}\right)=5,8,9,11 \text { or } 12[23]\end{array}$ \\
\hline Cycles & $\begin{array}{c}\text { For } n \geq 3, \lambda_{0,1}\left(C_{n}\right)=1 \text { or } 2[16] \\
\text { For } n \geq 3, \lambda_{1,1}\left(C_{n}\right)=2 \text { or } 3[21] \\
\text { For } n \geq 3, \lambda_{2,1}\left(C_{n}\right)=4[1] \\
\text { For, } n \geq 3, \lambda_{3,2,1}\left(C_{n}\right)=6,7,8 \text { or } 9[22] \\
\text { For, } n \geq 4, d \geq 5, \lambda_{d, 2,1}\left(C_{n}\right)=d+4, d+6,2 d+1 \\
\text { or } 2 d[22] \\
\text { For, } n \geq 3, \lambda_{4,3,2,1}\left(C_{n}\right)=9,11,14 \text { or } 13[23]\end{array}$ \\
\hline Complete & $\begin{array}{c}\lambda_{1,1}\left(k_{n}\right)=n-1[24] \\
\lambda_{d, 2,1}\left(K_{n}\right)=d(n-1)+1[22]\end{array}$ \\
\hline $\begin{array}{l}\text { Complete } \\
\text { bipartite }\end{array}$ & $\begin{array}{c}\lambda_{1,1}\left(K_{m, n}\right)=m+n-1[24] \\
\lambda_{d, 2,1}\left(K_{m, n}\right)=d+2(m+n)-3[22]\end{array}$ \\
\hline Planar & $\begin{array}{c}\lambda_{1,1}(G) \leq\lceil(5 / 3) \Delta+1\rceil+77[25] \\
\lambda_{2,1}(G) \leq 2 \Delta+35[26] \\
\lambda_{2,1}(G) \leq(5 / 3) \Delta+95[25] \\
\lambda_{h, k}(G) \leq k\lceil(5 / 3) \Delta\rceil+18 h+77 k-18[25] \\
\lambda_{3,2,1}(G) \leq 15\left(\Delta^{2}-\Delta+1\right)[27]\end{array}$ \\
\hline Interval & $\begin{array}{c}\lambda_{2,1}(G) \leq \Delta+w[28] \\
\lambda_{h, k}(G) \leq \max \cdot\{h, 2 k\} \Delta[29] \\
\lambda_{3,2,1}(G) \leq 6 \Delta-3[30] \\
\lambda_{4,3,2,1}(G) \leq 10 \Delta-6[30]\end{array}$ \\
\hline Circular-arc & $\begin{array}{c}\lambda_{0,1}(G) \leq \Delta[31] \\
\lambda_{1,1}(G) \leq 2 \Delta[31] \\
\lambda_{h, k}(G) \leq \max \cdot\{h, 2 k\} \Delta+h \omega[29] \\
\lambda_{2,1}(G) \leq \Delta+3 w[32] \\
\lambda_{3,2,1}(G) \leq 9 \Delta-6[33] \\
\lambda_{4,3,2,1}(G) \leq 16 \Delta-12[33]\end{array}$ \\
\hline Permutation & $\begin{array}{c}\lambda_{0,1}(G) \leq 2 \Delta-2[34] \\
\lambda_{0,1}(G) \leq \Delta-1[35] \\
\lambda_{2,1}(G) \leq \max \{4 \Delta-2,5 \Delta-8\}[36] \\
\lambda_{2,1}(G) \leq 5 \Delta-2[34]\end{array}$ \\
\hline
\end{tabular}

\section{Preliminaries and Notations}

A path is a graph $G=(V, E)$, where $\left(v_{j}, v_{j+1}\right) \in E$, for all $1 \leq j \leq n-1$, where $V=\left\{v_{1}, v_{2}, \ldots, v_{n}\right\}$, and it is denoted by $P_{n}$. Here, we consider IG (IG) which is not a path, so $\Delta>2$, because if $\Delta=2$, then it may be a path.

Let the set of intervals in real line be $I=\left\{I_{1}, I_{2}, \ldots, I_{n}\right\}$, where $I_{k}=\left[l_{k}, r_{k}\right], k=1,2, \ldots, n, l_{k}$, and $r_{k}$ are the left and right endpoints of $I_{k}$. For any interval $I_{k}, k=1,2, \ldots, n$, we draw a node $v_{k}$, and two nodes $v_{p}$ and $v_{q}$ have joined by a line segment that if the corresponding intervals have common portion, then we obtain an IG [44]. Throughout the paper, an interval $I_{k}$ and a node $v_{k}$ are the same. An IG and its interval representation are shown in Figure 3.

Notations. For any IG $G$ with $n$ nodes and corresponding set of intervals $I=\left\{I_{1}, I_{2}, \ldots, I_{n}\right\}$, we define the following: 

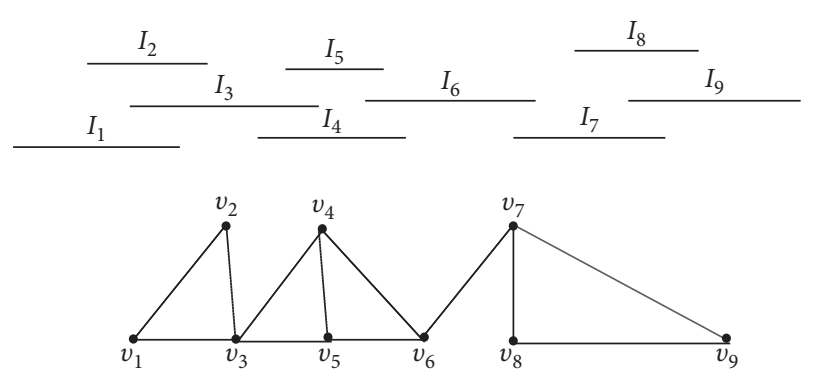

FIGURE 3: An interval representation and its corresponding IG.

(1) $L^{s}\left(I_{k}\right)$ : the set of used SL21-labels which are used before labelling the interval $I_{k}$, for every interval $I_{k} \in I$.

(2) $L^{i}\left(I_{k}\right)$ : the set of used SL21-labels at distance $i$ ( $i=$ $1,2)$ from the interval $I_{k}$, before labelling $I_{k}$, for every $I_{k} \in I$.

(3) $L^{\mathrm{vl}}\left(1, I_{k}\right)$ : the set of valid labels for the interval $I_{k}$ before labelling $I_{k}$, satisfying the adjoining condition of $L(2,1)$-labelling, for every interval $I_{k} \in I$.

(4) $L^{\mathrm{vl}}\left(2, I_{k}\right)$ : the valid set of labels of the interval $I_{k}$ before labelling $I_{k}$, satisfying $L(2,1)$-labelling condition, for every interval $I_{k} \in I$.

(5) $L^{\mathrm{svl}}\left(I_{k}\right)$ : the set of valid SL21-labels of the interval $I_{k}$ before labelling $I_{k}$, for every interval $I_{k} \in I$.

(6) $\lambda_{2,1}(G): L(2,1)$-labelling number of $G$.

(7) $f_{j}^{s}$ : the SL21-label of the interval $I_{j} \in I$.

(8) $L^{s}$ : the set of labels after completion of SL21-labelling of $G$.

\section{Surjective $L(2,1)$-Labelling of Paths}

In this portion, we have presented SL21-labelling of path and have showed that any path $P_{n}$ is surjectively $L(2,1)$-labelled if $n \geq 4$. Also, we have presented a greedy algorithm to label a path by SL21-labelling.

Theorem 1. For $P_{n}$,

$$
\lambda_{2,1}\left(P_{n}\right)= \begin{cases}1, & \text { if } n=1, \\ 3, & \text { if } n=2, \\ 4, & \text { if } n=3\end{cases}
$$

Proof. Let $P_{n}$ be a path having $n$ nodes.

Case $1: n=1$.

This result holds trivially.

Case 2: $n=2$.

The labels used are 1 and 3 and hence $\lambda_{2,1}\left(P_{2}\right)=3$. Case 3: $n=3$.

There are two possible cases shown in Figures 4(a) and 4(b). The labelling sequences are $\{3,1,4\}$ and $\{1,4,2\}$.
From the above result, it is concluded that $P_{n}$ can be SL21-labelled for $n=1,2,3$.

Theorem 2. The minimum path that can be labelled by SL21-labelling is $P_{4}$.

Proof. From Theorem 1, we have $\lambda_{2,1}\left(P_{2}\right)=3$ and $\lambda_{2,1}\left(P_{3}\right)=4$, so, for $n<4$, a path $P_{n}$ cannot be labelled by SL21-labelling. The labelling pattern $\{3,1,4,2\}$ of path $P_{4}$ (see Figure 5) shows that $P_{4}$ can be labelled by SL21-labelling. Hence, $P_{4}$ is the minimum path that can be labelled by SL21-labelling (Figure 6).

For this path, the node $V=\left\{v_{1}, v_{2}, \ldots, v_{22}\right\}$. Here, $n>4$, so this path can be surjectively labelled by $L(2,1)$-labelling. $f_{k}^{s}$ is the SL21-label of the node $v_{k}$, for $k=1,2, \ldots, 22$.

According to Algorithm 1, we rearrange the nodes as follows:

$v_{2}=v_{3}, \quad v_{3}=v_{5}, \quad v_{4}=v_{7}, \quad v_{5}=v_{9}, \quad v_{6}=v_{11}, \quad v_{7}=v_{13}$, $v_{8}=v_{15}, \quad v_{9}=v_{17}, \quad v_{10}=v_{19}, \quad v_{11}=v_{21}, \quad v_{12}=v_{4}, \quad v_{13}=v_{6}$, $v_{14}=v_{8}, v_{15}=v_{10}, v_{16}=v_{12}, v_{17}=v_{14}, v_{18}=v_{16}, v_{19}=v_{18}$, $v_{20}=v_{20}, v_{21}=v_{22}, v_{22}=v_{2}$, and $v_{1}$ remains unchanged.

Now, node $v_{k}$ is labelled by $k$; that is, $f_{k}^{s}=k$, for each $k=1,2, \ldots, 22$. After completion of surjective $L(2,1)$-labelling of $P_{22}$, the node and the label of the corresponding node are shown in Figure 7(b).

\section{Surjective $L(2,1)$-Labelling of IGs}

Here, some lemmas that we have used to develop the proposed algorithm are presented.

Lemma 1. For any $I G G,\left|L^{2}\left(I_{k}\right)\right| \leq \Delta-1$, for $I_{k} \in I$.

Proof. Let $G$ be an IG having $n$ nodes. The labelling of $G$ starts from the leftmost interval. Let node $v_{k}$ be corresponding to the interval $I_{k}$ of the IGG. Suppose that in a stage the intervals $I_{1}, I_{2}, \ldots, I_{k-1}$ (for some $k=2,3,4, \ldots, n$ ) are previously labelled by SL21-labelling and the remaining intervals are unlabelled.

Let $\left|L^{2}\left(I_{k}\right)\right|=p$. This means that the number of distinct SL21-labels used for labelling distance two intervals from the interval $I_{k}$ before labelling $I_{k}$ is $p$. Since the degree of the IG $G$ is $\Delta$, there exists an interval $I_{\alpha}$ (see Figure 8) and those are adjoining to $\Delta$ intervals at most. In Figure $8, I_{\alpha}$ is adjoining to $I_{k}, I_{\beta}, I_{k_{21}}, I_{k_{22}}$. Among the intervals, some intervals $\left(I_{\beta}, I_{k_{21}}, I_{k_{22}}\right.$ in Figure 8$)$ are of distance two apart from $I_{k}$ and among the intervals there is an interval $\left(I_{k}\right.$ in Figure 8) whose distance is not two from $I_{k}$. Hence, $p \leq \Delta-1$; that is, $\left|L^{2}\left(I_{k}\right)\right| \leq \Delta-1$.

Observation 1. For any IG $G, L^{i}\left(I_{k}\right) \subseteq L^{s}\left(I_{k}\right)$, for any interval $I_{k}, i=1,2$.

Observation 2. For any IG $G,\left|L^{1}\left(I_{k}\right)\right| \leq \Delta$, for any interval $I_{k} \in I$. 


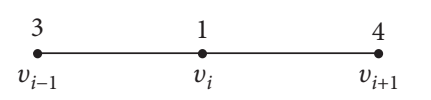

(a)

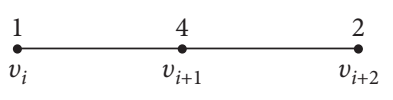

(b)

FIGURE 4: A path with three nodes and their surjective labels.

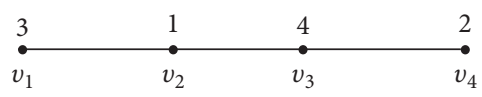

Figure 5: A path $P_{4}$ labelled by SL21-labelling.

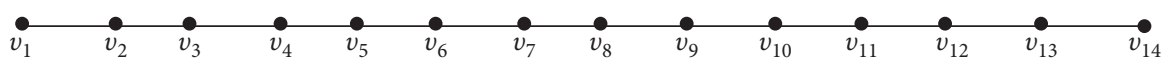

(a)

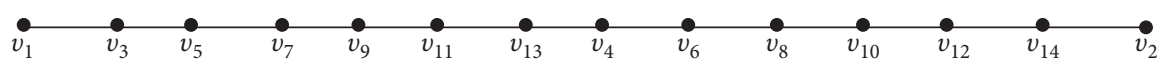

(b)

Figure 6: (a) A path with of 14 nodes; (b) the path after rearrangement of the nodes.

Input: The nodes of the path $P_{n}(n>6), V=\left\{v_{1}, v_{2}, \ldots, v_{n}\right\}$.

Output: The SL21-label of the path $P_{n}$.

Step 1: Rearrange the intervals as follows:

Case I: $n$ is odd

$v_{n}=v_{2}$

$v_{i+1}=v_{2 i+1}$, for $i=1,2, \ldots,((n-1) / 2)$;

$v_{i+((n-1) / 2)}=v_{2 i}$, for $i=2,3, \ldots,((n-1) / 2)$;

$v_{1}$ remains same;

Case II: $n$ is even

$v_{n}=v_{2}$

$v_{i+1}=v_{2 i+1}$, for $i=1,2, \ldots,(n / 2)-1$;

$v_{i+(n / 2)-1}=v_{2 i}$, for $i=2,3, \ldots,(n / 2)$;

$v_{1}$ remain same;

Step 2: Label the node $v_{i}$ by $i$, i.e., $f_{i}^{s}=i$, for $i=1,2, \ldots, n$ end AMPSL21.

Algorithm 1: AMPSPL21.

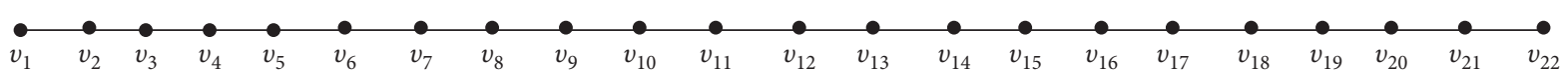

(a)

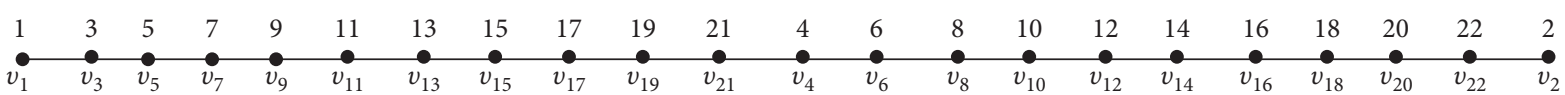

(b)

FIgURE 7: A path labeled by SL21-labelling.

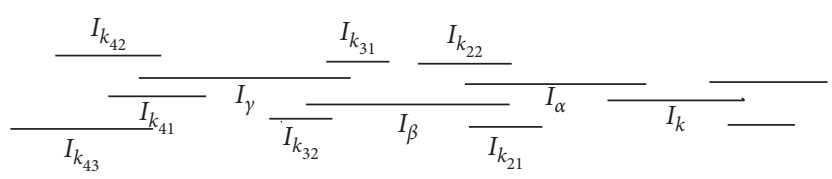

FIgURE 8: A set of intervals. 
Theorem 4. Any IGG with $n$ nodes is surjectively $L(2,1)$-labelled if $n=3 \Delta-1$.

Proof. Since $G$ has $n$ nodes, let $I=\left\{I_{1}, I_{2}, \ldots, I_{n}\right\}$. Since we want to label the intervals of an IG by SL21-labelling, every label is used exactly once and the labels must be in $\{1,2, \ldots, n\}$. So,

$$
\begin{aligned}
\lambda_{2,1}(G) & \leq 2\left|L^{1}\left(I_{k}\right)\right|+\left|L^{2}\left(I_{k}\right)\right| \\
& \leq 2 \Delta+(\Delta-1),[\text { by Lemmal }] \\
& \leq 3 \Delta-1 .
\end{aligned}
$$

Again, since $G$ has $n$ nodes, to label the whole graph by SL21-labelling, $n$ distinct labels must be required. Also, since $\lambda_{2,1}(G) \leq 3 \Delta-1$, in the extreme unfavorable cases $3 \Delta-1$ labels are required to label graph $G$ by $L(2,1)$-labelling. Again, in SL21-labelling, the highest label is equal to $n$. Hence, an IG $G$ is surjectively labelled using $L(2,1)$-labelling if $n=3 \Delta-1$.

If $n \neq 3 \Delta-1$, then the IG may or may not be labelled by SL21-labelling, because in the worst case $3 \Delta-1$ labels are required to label the IG, which is not equal to $n$. This contradicts the condition that the used label must belong to $\{1,2, \ldots, n\}$ and the highest label must be equal to $n$ for SL21-labelling.

4.1. Algorithm for Surjective L(2,1)-Labelling of IGs. In this part, two algorithms are designed: one is to compute $L^{\mathrm{vl}}\left(k, I_{j}\right)$ and the other is to compute SL21-label for an IG (Algorithm 2).

Lemma 2. $L^{v l}\left(p, I_{k}\right)$ for $p=1,2$ is correctly computed by Algorithm 2 and the time complexity of the above algorithm is $O\left(\Delta^{2}\right)$.

Proof. According to Algorithm 2, each element $i \in L^{\mathrm{vl}}\left(1, I_{j}\right)$ differs from $l_{r}$ by at least 2 for each $l_{r} \in L^{1}\left(I_{k}\right)$. Therefore, $\mid i-$ $l_{r} \mid \geq 2$ for all $i \in L^{\mathrm{vl}}\left(1, I_{k}\right)$ and for all $l_{r} \in L^{1}\left(I_{k}\right)$. So, Algorithm 2 correctly computes the set $L^{\mathrm{vl}}\left(1, I_{k}\right)$ for each $I_{k} \in I$, $k=2,3, \ldots, n$. Again, each element $l_{\alpha}$ of $L^{\mathrm{vl}}\left(2, I_{k}\right)$ differs from $l_{\beta}$ by at least 1 for each $l_{\beta} \in L^{2}\left(I_{k}\right)$. Therefore, $\left|l_{m}-p_{n}\right| \geq 2$ for all $l_{m} \in L^{\mathrm{vl}}\left(2, I_{k}\right)$ and for all $p_{n} \in L^{1}\left(I_{k}\right)$, and $\left|l_{m}-p_{n}\right| \geq 1$ for all $l_{m} \in L^{\mathrm{vl}}\left(2, I_{k}\right)$ and for all $p_{n} \in L^{2}\left(I_{k}\right)$. Therefore, Algorithm 2 correctly computes $L^{\mathrm{vl}}\left(p, I_{k}\right)$ for every $k=1,2$. As $\left|L^{s}\right|$ is the cardinality of the set of labels $L^{s},\left|L^{i}\left(I_{k}\right)\right| \leq\left|L^{s}\right|$ for $i=1,2$ and $I_{k} \in I$, and also $r \leq 3 \Delta+1$, where $r=\max \left\{L^{s}\left(I_{k}\right)\right\}+2$. So, $L^{\mathrm{vl}}\left(1, I_{k}\right)$ is computed by using at most $(3 \Delta+1)\left|L^{s}\right|$ times, that is, using $O\left(\Delta\left|L^{s}\right|\right)$ times. Again, $\left|L^{\mathrm{vl}}\left(2, I_{n}\right)\right| \leq(3 \Delta+1)$, so, $L^{\mathrm{vl}}\left(2, I_{k}\right)$ is computed using at most $(3 \Delta+1)\left|L^{s}\right|$ times, that is, using $O\left(\Delta\left|L^{s}\right|\right)$ times. Since $\left|L^{s}\right| \leq 3 \Delta+1$, the iterative time for algorithm SLKVL is $O\left(\Delta^{2}\right)$.

Lemma 3. For each $I G G, L^{v l}\left(1, I_{k}\right)$ is the nonempty largest set satisfying distance one condition of $L(2,1)$-labelling, $l \leq r$, for every $l \in L^{v l}\left(1, I_{k}\right)$, and $r=\max \left\{L^{s}\left(I_{k}\right)\right\}+2$, for any $I_{k} \in I$.
Proof. Since $r=\max \left\{L^{s}\left(I_{k}\right)\right\}+2$ and $L^{1}\left(I_{k}\right) \subseteq L^{s}\left(I_{k}\right)$ (by Observation 1$),\left|r-l_{i}\right| \geq 2$ for every $l_{i} \in L^{1}\left(I_{k}\right)$. Therefore, $r \in L^{\mathrm{vl}}\left(1, I_{k}\right)$, so, $L^{\mathrm{vl}}\left(1, I_{k}\right)$ is a nonempty set. Also, let $B$ be an arbitrary set of labels, which satisfies distance one condition of $L(2,1)$-labelling, $l \leq r$, for all $l \in B$, and $r=\max \left\{L^{s}\left(I_{k}\right)\right\}+2$. Then, for $b \in B,\left|b-l_{i}\right| \geq 2$ for any $l_{i} \in L^{1}\left(I_{k}\right)$. Thus, $b \in L^{\mathrm{vl}}\left(1, I_{k}\right)$. So, $b \in B \Rightarrow b \in L^{\mathrm{vl}}\left(1, I_{k}\right)$. Then, $B \subseteq L^{\mathrm{vl}}\left(1, I_{k}\right)$. Since $B$ is arbitrary, $L^{\mathrm{vl}}\left(1, I_{k}\right)$ is the largest nonempty set of labels which satisfies distance one condition of $L(2,1)$-labelling, $l \leq r$, for every $l \in L^{\mathrm{vl}}\left(1, I_{k}\right)$, and $r=\max \left\{L^{s}\left(I_{k}\right)\right\}+2$, for any $I_{k} \in I$.

Lemma 4. For any $I G G, L^{v l}\left(2, I_{k}\right)$ is the nonempty largest set satisfying $L(2,1)$-labelling condition, $l \leq r$, for every $l \in L^{v l}\left(1, I_{k}\right), r=\max \left\{L^{s}\left(I_{k}\right)\right\}+2$, and $I_{k} \in I$.

Proof. Since $r=\max \left\{L^{s}\left(I_{k}\right)\right\}+2$ and $L^{i}\left(I_{k}\right) \subseteq L^{s}\left(I_{k}\right)$, for $i=$ 1,2 (by Observation 1 ), $\left|r-l_{p}\right| \geq 2$ for $l_{p} \in L^{i}\left(I_{k}\right), i=1,2$; that is, $\left|r-l_{p}\right| \geq 2$ for all $l_{p} \in L^{1}\left(I_{k}\right)$ and $\left|r-l_{p}\right| \geq 1$ for all $l_{p} \in L^{2}\left(I_{k}\right)$. Hence, $r$ is the valid $L(2,1)$-label of $I_{k}$; therefore, $r \in L^{\mathrm{vl}}\left(2, I_{k}\right)$. This shows that $L^{\mathrm{vl}}\left(2, I_{k}\right)$ is a nonempty set. Also, let $B$ be an arbitrary set of labels which satisfies $L(2,1)$-labelling conditions, $l \leq r$ for every $l \in B$, and $r=\max \left\{L^{s}\left(I_{k}\right)\right\}+2$. Then, for $b \in B,\left|b-l_{p}\right| \geq 2$ for $l_{p} \in L^{1}\left(I_{k}\right)$ and $\left|b-l_{q}\right| \geq 1$ for any $l_{q} \in L^{2}\left(I_{k}\right)$. Thus, $b \in L^{\mathrm{vl}}\left(2, I_{k}\right)$. Thus, $b \in B \Rightarrow b \in L^{\mathrm{vl}}\left(2, I_{K}\right)$. So, $B \subseteq L^{\mathrm{vl}}\left(2, I_{k}\right)$. Since $B$ is arbitrary, $L^{\mathrm{vl}}\left(2, I_{k}\right)$ is the largest nonempty set of labels which satisfies $L(2,1)$-labelling, $\quad l \leq r$ for every $l \in L^{\mathrm{vl}}\left(2, I_{k}\right)$, and $r=\max \left\{L^{s}\left(I_{k}\right)\right\}+2$, for any $I_{k} \in I$ (Algorithm 3 ).

Theorem 5. Algorithm 3 correctly labels an $I G$ by SL21-labelling, where $n=3 \Delta-1$.

Proof. Let $G$ be an IG with $n$ nodes such that $n=3 \Delta-1$. We rearranged the nodes so that no two consecutive intervals are adjacent to each other. After rearrangement of the intervals, let $I=\left\{I_{1}, I_{2}, \ldots, I_{n}\right\}$ and let $f_{1}^{s}=1$ and $L^{s}\left(I_{2}\right)=\{1\}$.

We consider circumstances in which the intervals $I_{1}, I_{2}, \ldots, I_{k-1}$ are already labelled for $k=2,3, \ldots, n$ and the remaining intervals are not labelled. In this stage, our aim is to label $I_{k}$ by SL21-labelling. Now, $L^{\mathrm{vl}}\left(2, I_{k}\right)$ is the nonempty largest set of labels satisfying $L(2,1)$-labelling, $l \leq r$ for any $l \in L^{\mathrm{vl}}\left(2, I_{k}\right)$, and $r=\max \left\{L^{s}\left(I_{k}\right)\right\}+2$ for every $I_{k} \in I$ (by Lemma 4).

Again, $L^{\text {svl }}\left(I_{k}\right)=L^{\mathrm{vl}}\left(2, I_{k}\right)-L^{s}\left(I_{k}\right)$, so $L^{\text {svl }}\left(I_{k}\right)$ is the nonempty largest set satisfying SL21-labelling, as the label in $L^{\text {svl }}\left(I_{k}\right)$ was not used previously to label any interval and also satisfies $L(2,1)$-labelling. Therefore, $f_{k}^{s}=q$, where $q=\min \left\{L^{\text {svl }}\left(2, I_{k}\right)\right\}$. Since $L^{\text {svl }}\left(I_{k}\right)$ is the largest set of labels satisfying SL21-labelling, $q$ is the least surjective label of $I_{k}$. Since $\lambda_{2,1}(G) \leq 3 \Delta-1$, the label of $I_{k}$ must be less than or equal to $3 \Delta-1$. Again, since $n=3 \Delta-1$, the interval $I_{k}$ is labelled by using only the labels from $\{1,2, \ldots, n\}$ which have not been used earlier to label any interval. Since $I_{k}$ is arbitrary, any IG is surjectively labelled by $L(2,1)$-labelling by Algorithm 3. 
Input: $I_{k}, L^{1}\left(I_{k}\right), L^{2}\left(I_{k}\right)$ and $L^{s}\left(I_{k}\right), k=2,3, \ldots, n$.

Output: $L^{\mathrm{vl}}\left(p, I_{k}\right)$ for $p=1,2 ; k=2,3, \ldots, n$.

Step 1: for $i=1$ to $r$, where $r=\max \left\{L^{s}\left(I_{k}\right)\right\}+2$

for $j=1$ to $\left|L^{1}\left(I_{k}\right)\right|$

let $l_{j}$ be the $j$ th element of $L^{1}\left(I_{k}\right)$

if $\left|i-l_{j}\right| \geq 2$, then add $i$ to the set $L^{\mathrm{vl}}\left(1, I_{k}\right)$;

end for;

end for;

Step 2: for $m=1$ to $\left|L^{\mathrm{vl}}\left(1, I_{k}\right)\right|$

for $n=1$ to $\left|L^{2}\left(I_{k}\right)\right|$

$l_{m}$ and $p_{n}$ be the elements of $\left|L^{\mathrm{vl}}\left(1, I_{k}\right)\right|$ and $\left|L^{2}\left(I_{k}\right)\right|$ respectively;

if $\left|l_{m}-p_{n}\right| \geq 1$, then add $l_{m}$ to the set $L^{\mathrm{vl}}\left(2, I_{k}\right)$;

end for;

end for;

end AMPKVL.

Algorithm 2: AMPKVL.

Input: The intervals of an IG $I=\left\{I_{1}, I_{2}, \ldots, I_{n}\right\}$ and $L^{\mathrm{vl}}\left(p, I_{k}\right)$ for $k=2,3, \ldots, n$ and $p=1,2$ where $n=3 \Delta-1$.

Output: $f_{k}^{s}$, the SL21-label of the interval $I_{k}, k=1,2, \ldots, n$.

Step 1: Rearrange the intervals as follows:

Case I: $n$ is odd

$I_{n}=I_{2}$;

$I_{i+1}=I_{2 i+1}$, for $i=1,2, \ldots,((n-1) / 2)$;

$I_{i+((n-1) / 2)}=I_{2 i}$, for $i=2,3, \ldots,((n-1) / 2)$;

$I_{1}$ remains same;

Case II: $n$ is even

$I_{n}=I_{2}$

$I_{i+1}=I_{2 i+1}$, for $i=1,2, \ldots,(n / 2)-1$;

$I_{i+(n / 2)-1}=I_{2 i}$, for $i=2,3, \ldots,(n / 2)$;

$I_{1}$ is unchanged;

Step 2: (Initialization)

$f_{1}^{s}=1$;

$L^{s}\left(I_{2}\right)=\{1\}$

Step 3: for $k=2$ to $n-1$

$L^{\mathrm{svl}}\left(I_{k}\right)=L^{\mathrm{vl}}\left(2, I_{k}\right)-L^{s}\left(I_{k}\right)$;

$f_{k}^{s}=\min \left\{L^{\mathrm{svl}}\left(I_{k}\right)\right\}$;

$L^{s}\left(I_{k+1}\right)=L^{s}\left(I_{k}\right) \bigcup\left\{f_{k}^{s}\right\}$

end for;

Step 4: $L^{\text {svl }}\left(I_{n}\right)=L^{\mathrm{vl}}\left(2, I_{n}\right)-L^{s}\left(I_{n}\right)$;

$f_{n}^{s}=\min \left\{L^{\mathrm{svl}}\left(I_{n}\right)\right\}$

Step 5: $L^{s}=L^{s}\left(I_{n}\right) \bigcup\left\{f_{n}^{s}\right\}$;

end AMPSL 21 .

Algorithm 3: AMPSL21.

Theorem 6. The running time of Algorithm 3 is $O\left(n \Delta^{3}\right)$, where $n=3 \Delta-1$.

Proof. According to Algorithm 3, the SL21-label of $I_{k}$, that is, $f_{k}^{s}$, is computed if the set $L^{\text {svl }}\left(I_{k}\right)$ is computed. Now by Lemma 2 we see that by algorithm AMPSL21 one can compute the set $L^{\mathrm{vl}}\left(p, I_{k}\right), p=1,2$, by using $O\left(\Delta^{2}\right)$ time. Again, by Appendix, algorithm $A \operatorname{diff} B, L^{\mathrm{vl}}\left(2, I_{k}\right)-L^{s}\left(I_{k}\right)$ have been computed in $O(\Delta)$ time, for any $k$. Therefore, the time needed for computing $L^{\text {svl }}\left(I_{k}\right)$ is $O\left(\Delta^{3}\right)$. Since we need to find $L^{\text {svl }}\left(2, I_{k}\right)$ for $k=2,3, \ldots, n$, the time complexity for Algorithm 3 is $O\left((n-1) \Delta^{3}\right)$, that is, $O\left(n \Delta^{3}\right)$.

4.1.1. Illustration of Algorithm AMPSL21. We take an IG having 14 nodes (see Figure 9) and label that graph by Algorithm 3. The graph after completion of surjective $L(2,1)$-labelling is given in Figure 10 .

For the above graph, the set of intervals $I=\left\{I_{1}, I_{2}\right.$, $\left.\ldots, I_{14}\right\}$ and $\Delta=5$. Here, $3 \Delta-1=14=n$, so this IG can be 


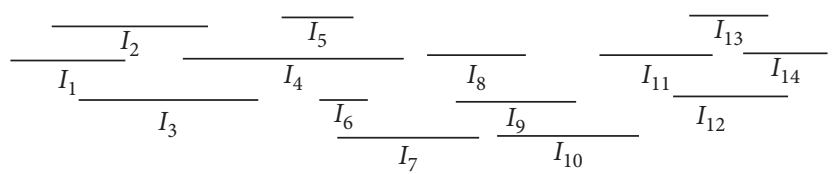

Figure 9: AnIG.

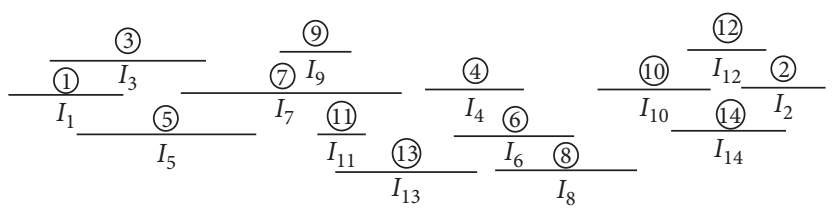

FIgURE 10: An IG labelled by SL21-labelling.

TABle 2: Nodes and their corresponding surjective L(2,1)-labels.

\begin{tabular}{|c|c|c|c|c|c|c|c|c|c|c|c|c|c|c|}
\hline Nodes & $v_{1}$ & $v_{2}$ & $v_{3}$ & $v_{4}$ & $v_{5}$ & $v_{6}$ & $v_{7}$ & $v_{8}$ & $v_{9}$ & $v_{10}$ & $v_{11}$ & $v_{12}$ & $v_{13}$ & $v_{14}$ \\
\hline Surjective $L(2,1)$-labels & 1 & 2 & 3 & 4 & 5 & 6 & 7 & 8 & 9 & 10 & 11 & 12 & 13 & 14 \\
\hline
\end{tabular}

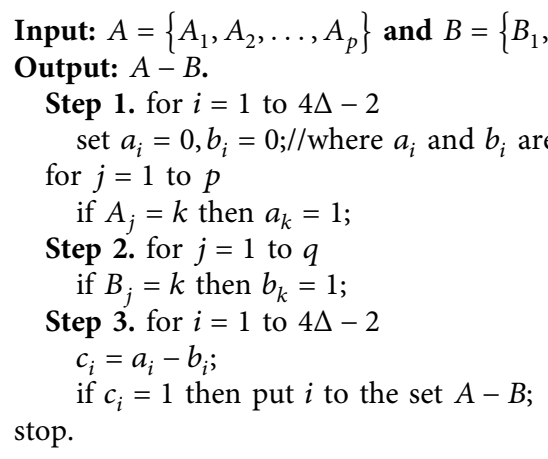

Algorithm 4: $A$ diff $B$

surjectively labelled by $L(2,1)$-labelling. $f_{k}^{s}$ is the SL21-label of the interval $I_{k}$, for $k=1,2, \ldots, 14$.

According to Algorithm 3, at first, we rearrange the intervals as follows:

$$
I_{2}=I_{3}, \quad I_{3}=I_{5}, \quad I_{4}=I_{7}, \quad I_{5}=I_{9}, \quad I_{6}=I_{11}, \quad I_{7}=I_{13},
$$
$I_{8}=I_{4}, \quad I_{9}=I_{6}, \quad I_{10}=I_{8}, \quad I_{11}=I_{10}, \quad I_{12}=I_{12}, \quad I_{13}=I_{14}$, $I_{14}=I_{2}$, and $I_{1}$ remains the same.

$f_{1}^{s}=1$ and $L^{s}\left(I_{2}\right)=\{1\}$ are also initialized.

Iteration 1: For $k=2$,

$$
\begin{aligned}
& L^{1}\left(I_{2}\right)=\{1\}, L^{2}\left(I_{2}\right)=\phi . \\
& L^{\mathrm{vl}}\left(1, I_{2}\right)=\{1,2,3\}, L^{\mathrm{vl}}\left(2, I_{2}\right)=\{1,2,3\} .
\end{aligned}
$$$$
\text { So, } \quad L^{\text {svl }}\left(I_{2}\right)=L^{\mathrm{vl}}\left(2, I_{2}\right)-L^{s}\left(I_{2}\right)=\{1,2,3\}-\{1\}=
$$$$
\{2,3\} \text {. }
$$

Therefore, $f_{2}^{s}=\min \left\{L^{\mathrm{svl}}\left(I_{2}\right)\right\}=2$.

$$
L^{s}\left(I_{3}\right)=L^{s}\left(I_{2}\right) \cup\left\{f_{2}^{s}\right\}=\{1\} \cup\{2\}=\{1,2\} .
$$

Iteration 2: For $k=3$,

$$
\begin{aligned}
& L^{1}\left(I_{3}\right)=\{1\}, L^{2}\left(I_{3}\right)=\phi . \\
& L^{\mathrm{vl}}\left(1, I_{3}\right)=\{3,4\}, L^{\mathrm{vl}}\left(2, I_{3}\right)=\{3,4\} .
\end{aligned}
$$

So, $\quad L^{\text {svl }}\left(I_{3}\right)=L^{\mathrm{vl}}\left(2, I_{3}\right)-L^{s}\left(I_{3}\right)=\{3,4\}-\{1,2\}$

$=\{3,4\}$.

Therefore, $f_{3}^{s}=\min \left\{L^{\text {svl }}\left(I_{3}\right)\right\}=3$.

$L^{s}\left(I_{4}\right)=L^{s}\left(I_{3}\right) \cup\left\{f_{3}^{s}\right\}=\{1,2,3\}$.

\section{Iteration 3: For $k=4$,}

$L^{1}\left(I_{4}\right)=\phi, L^{2}\left(I_{4}\right)=\phi$.

$L^{\mathrm{vl}}\left(1, I_{4}\right)=\{1,2,3,4,5\}, L^{\mathrm{vl}}\left(2, I_{4}\right)=\{1,2,3,4,5\}$.

So, $L^{\mathrm{svl}}\left(I_{4}\right)=L^{\mathrm{vl}}\left(2, I_{4}\right)-L^{s}\left(I_{4}\right)=$

$\{1,2,3,4,5\}-\{1,2,3\}=\{4,5\}$.

Therefore, $f_{4}^{s}=\min \left\{L^{\text {svl }}\left(I_{4}\right)\right\}=4$.

$L^{s}\left(I_{5}\right)=L^{s}\left(I_{4}\right) \cup\left\{f_{4}^{s}\right\}=\{1,2,3,4\}$.

Iteration 4: For $k=5$,

$L^{1}\left(I_{5}\right)=\{1,3\}, L^{2}\left(I_{5}\right)=\phi$.

$L^{\mathrm{vl}}\left(1, I_{5}\right)=\{5,6\}, L^{\mathrm{vl}}\left(2, I_{5}\right)=\{5,6\}$.

So,

$\{5,6\}-\{1,2,3,4\}=\{5,6\}$.

$$
L^{\mathrm{svl}}\left(I_{5}\right)=L^{\mathrm{vl}}\left(2, I_{5}\right)-L^{s}\left(I_{5}\right)=
$$

Therefore, $f_{5}^{s}=\min \left\{L^{\text {svl }}\left(I_{5}\right)\right\}=5$.

$L^{s}\left(I_{6}\right)=L^{s}\left(I_{5}\right) \cup\left\{f_{5}^{s}\right\}=\{1,2,3,4,5\}$. 


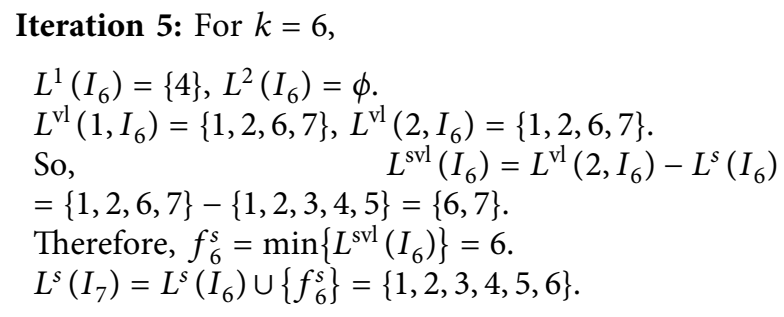

In this way, $f_{7}^{s}=7, f_{8}^{s}=8, f_{9}^{s}=9, f_{10}^{s}=10, f_{11}^{s}=11$, $f_{12}^{s}=12, f_{13}^{s}=13$, and, finally, $f_{14}^{s}=14$.

The nodes and their corresponding labels are shown in Table 2.

\section{Conclusion}

In $L(2,1)$-labelling, although there is a light chance to overlap the frequencies in radio network, it cannot be neglected, but in SL21-labelling there is no chance to overlap the frequencies, as in this case the labels are distinct. So, the results about SL21-labelling are clearly welcome. In the entire article, we have proved that a path $P_{n}$ can be surjectively $L(2,1)$-labelled if $n \geq 4$. An algorithm is also presented to label a path of $n$ nodes, for $n \geq 7$. Also, we have showed that an IGG having $n$ nodes can be surjectively labelled by $L(2,1)$-labelling if $n=3 \Delta-1$. Also, we have presented an $O\left(n \Delta^{3}\right)$ time algorithm to label an IG by SL21-labelling. If $n \neq 3 \Delta-1$, then the IG may or may not be labelled by SL21-labelling. In the future, we can extend this work to other classes of intersection graph. So, there is a scope for the new research to study surjective labelling of permutation graph, trapezoid graph, and so forth.

\section{Appendix}

Here, an algorithm to compute $A-B$ is presented, where $A$ and $B$ are subsets of $\{1,2, \ldots, 4 \Delta-2\}$ (Algorithm 4).

\section{Data Availability}

No data were used to support this study.

\section{Conflicts of Interest}

The authors declare that they have no conflicts of interest.

\section{Acknowledgments}

This work was supported by the Taif University Researchers Supporting Project (TURSP-2020/246), Taif University, Taif, Saudi Arabia.

\section{References}

[1] J. R. Griggs and R. K. Yeh, "Labelling graphs with a condition at distance 2," SIAM Journal on Discrete Mathematics, vol. 5, no. 4, pp. 586-595, 1992.

[2] S. Amanathulla and M. Pal, " $L(3,2,1)$-labelling problems on permutation graphs," Transylvanian Review, vol. 25, no. 14, pp. 3939-3953, 2017.
[3] S. Amanathulla and M. Pal, " $L\left(h_{1}, h_{2}, \ldots, h_{m}\right)$-labelling problems on interval graphs," International Journal of Control Theory and Applications, vol. 10, no. 1, pp. 467-479, 2017.

[4] S. Amanathulla and M. Pal, " $L\left(h_{1}, h_{2}, \ldots, h_{m}\right)$ labeling problems on circular-arc graphs," Far East Journal of Mathematical Sciences (FJMS), vol. 102, no. 6, pp. 1279-1300, 2017.

[5] S. Amanathulla and M. Pal, " $L(1,1,1)$ - and $L(1,1,1,1)$-labeling problems of square of path, complete graph and complete bipartite graph," Far East Journal of Mathematical Sciences (FJMS), vol. 106, no. 2, pp. 515-527, 2018.

[6] S. Amanathulla, M. Pal, and S. Sahoo, " $L(3,1,1)$-labeling numbers of square of paths, complete graphs and complete bipartite graphs," Journal of Intelligent \& Fuzzy Systems, vol. 36, no. 2, pp. 1917-1925, 2019.

[7] M. Berhe and C. Wang, "Computation of certain topological coindices of graphene sheet and $\mathrm{C} 4 \mathrm{C} 8(\mathrm{~S})$ nanotubes and nanotorus," Applied Mathematics and Nonlinear Sciences, vol. 4, no. 2, pp. 455-468, 2019.

[8] S. Goyal, P. Garg, and V. N. Mishra, "New composition of graphs and their Wiener Indices," Applied Mathematics and Non Linear Sciences, vol. 4, no. 1, pp. 175-180, 2019.

[9] S. M. Hosamani, V. B. Awati, and R. M. Honmore, "On graphs with equal dominating and c-dominating energy," Applied Mathematics and Nonlinear Sciences, vol. 4, no. 2, pp. 503512, 2019.

[10] B. M. Gurevich, "On classes of infinite loaded graphs with randomly deleted edges," Applied Mathematics and Nonlinear Sciences, vol. 5, no. 2, pp. 257-260, 2020.

[11] P. S. Ranjini, V. Lokesha, and S. Kumar, "Degree sequence of graph operator for some standard graphs," Applied Mathematics and Non Linear Sciences, vol. 5, no. 2, pp. 99-108, 2020.

[12] K. Jonas, Graph coloring analogues with a condition at distance two: l(2,1)-labellings and list $\lambda$-labellings, Ph.D. Thesis, University of South Carolina, Columbia, SC, USA, 1993.

[13] G. J. Chang and D. Kuo, "The $L(2,1)$-labeling problem on graphs," SIAM Journal on Discrete Mathematics, vol. 9, no. 2, pp. 309-316, 1996.

[14] D. Král' and R. Skrekovski, "A Theorem about the channel assignment problem," SIAM Journal on Discrete Mathematics, vol. 16, no. 3, pp. 426-437, 2003.

[15] D. Goncalves, "On the $L(d, 1)$-labellinng of graphs," Discrete Mathematics, vol. 308, pp. 1405-1414, 2008.

[16] A. A. Bertossi and M. A. Bonuccelli, "Code assignment for hidden terminal interference avoidance in multihop packet radio networks," IEEE/ACM Transactions on Networking, vol. 3, no. 4, pp. 441-449, 1995.

[17] R. K. Yeh, Labeling graphs with a condition at distance two, Ph.D Thesis, University of South Carolina, Columbia, C, USA, 1990.

[18] M. L. Chia, D. Qua, H. Liao, C. Yang, and R. K. Yea, “ $L(3,2,1)$ -labelling of graphs," Taiwanese Journal of Mathematics, vol. 15, no. 6, pp. 2439-2457, 2011.

[19] J. Clipperton, " $L(4,3,2,1)$-labelling of simple graphs," Applied Mathematics Science, vol. 1, no. 2, pp. 95-102, 2011.

[20] T. Makansi, "Transmitter-oriented code assignment for multihop packet radio," IEEE Transactions on Communications, vol. 35, no. 12, pp. 1379-1382, 1987.

[21] R. Battiti, A. A. Bertossi, and M. A. Bonuccelli, "Assigning codes in wireless networks: bounds and scaling properties," Wireless Networks, vol. 5, no. 3, pp. 195-209, 1999.

[22] J. Clipperton, " $L(\mathrm{~d}, 2,1)$-labelling of simple graphs," Math Journal, vol. 9, pp. 1-11, 2008. 
[23] S. Atta and P. R. S. Mahapatra, " $L(4,3,2,1)$-labelling for simple graphs," Informatation Systems Design and Intelligent Applications, vol. 339, pp. 89-105, 2015.

[24] J. P. Georges and D. W. Mauro, "Generalized vertex labelling with a condition at distance two," Congres. Numer.vol. 109, pp. 141-159, 1995.

[25] M. Molloy and M. R. Salavatipour, "A Bound on the chromatic number of the square of a planar graph," Journal of Combinatorial Theory, Series B, vol. 94, no. 2, pp. 189-213, 2005.

[26] J. van den Heuvel and S. McGuinness, "Coloring the square of a planar graph," Journal of Graph Theory, vol. 42, no. 2, pp. 110-124, 2003.

[27] J. Lui and Z. Shao, "The $\mathrm{L}(3,2,1)$-labelling problem on graphs," Mathematics Applicata, vol. 17, no. 4, pp. 596-602, 2004.

[28] S. Paul, M. Pal, and A. Pal, " $L(2,1)$-labelling of interval graph," Journal of Applied Mathematics and Computing, vol. 49, no. 1, pp. 419-432, 2015.

[29] T. Calamoneri, S. Caminiti, R. Petreschi, and S. Olariu, "On the $L(h, k)$-labeling of co-comparability graphs and circulararc graphs," Networks, vol. 53, no. 1, pp. 27-34, 2009.

[30] S. Amanathulla and M. Pal, " $L(3,2,1)$ - and $L(4,3,2,1)$ labeling problems on interval graphs," AKCE International Journal of Graphs and Combinatorics, vol. 14, no. 3, pp. 205-215, 2017.

[31] S. Amanathulla and M. Pal, " $L(0,1)$ - and $L(1,1)$-labelling problems on circular-arc graphs," International Journal of Soft Computing, vol. 11, no. 6, pp. 343-350, 2016.

[32] S. Paul, M. Pal, and A. Pal, " $L(2,1)$-labelling of circular-arc graph," Annals of Pure and Applied Mathematics, vol. 5, no. 2, pp. 208-219, 2014.

[33] S. Amanathulla and M. Pal, " $L(3,2,1)$ - and $L(4,3,2,1)$-labelling problems on circular-arc graphs," International Journal of Control Theory and Applications, vol. 9, no. 34, pp. 869-884, 2016.

[34] H. L. Bodlaender, T. Kloks, R. B. Tan, and J. V. Leeuwen, "Approximations for -colorings of graphs," The Computer Journal, vol. 47, no. 2, pp. 193-204, 2004.

[35] S. Paul, M. Pal, and A. Pal, " $L(0,1)$-labelling of permutation graphs," Journal of Mathematical Modelling and Algorithms in Operations Research, vol. 14, no. 4, pp. 469-479, 2015.

[36] S. Paul, M. Pal, and A. Pal, " $L(2,1)$-labeling of permutation and bipartite permutation graphs," Mathematics in Computer Science, vol. 9, no. 1, pp. 113-123, 2014.

[37] M. Lingscheit, K. Ruff, and J. Ward, " $L(d, j, s)$-minimal and surjective graph labelling-," Rose Hulman Undergragute Mathematics Journal, vol. 10, no. 2, pp. 1-27, 2009.

[38] S. Amanathulla and M. Pal, "Surjective $L(2,1)$-labeling of cycles and circular-arc graphs," Journal of Intelligent \& Fuzzy Systems, vol. 35, no. 1, pp. 739-748, 2018.

[39] H. Rashmanlou, G. Muhiuddin, S. Amanathulla, F. Mofidnakhaei, and M. Pal, "A study on cubic graphs with novel application," Journal of Intelligent \& Fuzzy Systems, vol. 40, no. 1, pp. 89-101, 2021.

[40] S. S. Hussain, R. J. Hussain, and G. Muhiuddin, "Neutrosophic vague line graphs," Neutrosophic Sets and Systems, vol. 36, pp. 121-130, 2020.

[41] G. Muhiuddin, M. Mohseni Takallo, Y. B. Jun, and R. A. Borzooei, "Cubic graphs and their application to a traffic flow problem," International Journal of Computational Intelligence Systems, vol. 13, no. 1, pp. 1265-1280, 2020.

[42] T. Pramanik, G. Muhiuddin, A. M. Alanazi, and M. Pal, “An extension of fuzzy competition graph and its uses in manufacturing industries," Mathematics, vol. 8, no. 6, p. 1008, 2020.

[43] S. Samanta, G. Muhiuddin, A. M. Alanazi, and K. Das, "Mathematical approach on representation of competitions: competition cluster hypergraphs," Mathematical Problems in Engineering, vol. 2020, Article ID 2517415, 10 pages, 2020.

[44] Sk. Amanathulla and M. Pal, " $L(\mathrm{~h}, \mathrm{k})$-labelling problems on intersection graphs," in Handbook of Research on Advanced Applications of Graph Theory in Modern Society, pp. 437-468, IGI Global, Hershey, PA, USA, 2020. 